\title{
EMPREGO DA ACUPUNTURA VETERINÁRIA NA DISPLASIA COXOFEMORAL EM CÃES
}

\author{
Leonardo Kouri Albuquerque', Yuri Karaccas de Carvalho² \\ ${ }^{1}$ Mestrando do Programa de Pós Graduação em Sanidade e Produção Animal na \\ Amazônia Ocidental, Universidade Federal do Acre - UFAC, Rio Branco - Acre \\ (leonardo_kouri@hotmail.com) \\ 2Professor Doutor Docente do curso de Medicina Veterinária da Universidade \\ Federal do Acre - UFAC
}

Recebido em: 02/10/2017 - Aprovado em: 21/11/2017 - Publicado em: 05/12/2017 DOI: 10.18677/EnciBio_2017B123

\begin{abstract}
RESUMO
O presente estudo visa reunir informações atualizadas a respeito do emprego da acupuntura veterinária na displasia coxofemoral em cães, identificar quais os métodos de acupuntura mais empregados e os principais acupontos utilizados na terapêutica desta patologia. Tendo em vista que a acupuntura se mostra uma ferramenta importante no restabelecimento da força muscular, controle da dor e inflamação, permitindo ao paciente, melhora das atividades locomotoras, aumento do ângulo de movimento da articulação e consequente melhoria da qualidade de vida, aliada a um melhor custo econômico, menor tempo de recuperação e redução dos riscos cirúrgicos e complicações pós-operatórias. Apesar da pouca quantidade de estudos direcionados ao emprego da acupuntura veterinária na displasia coxofemoral em cães, verificou-se que os acupontos locais da articulação coxofemoral são os mais empregados e permitem a indução dos processos de reparação de forma objetiva e efetiva, principalmente quando estimulados por meio dos métodos de acupuntura por agulhamento simples e implantes de ouro.
\end{abstract}

PALAVRAS-CHAVE: Caninos. Doenças articulares. Medicina Tradicional Chinesa.

\section{THE USE OF VETERINARY ACUPUNCTURE IN THE TREATMENT OF DOGS' HIP DYSPLASIA}

\begin{abstract}
The present report aims to collect updated data on the use of veterinary acupuncture in the treatment of dogs' hip dysplasia; the research, beside, intends to identify which are the most applied acupuncture methods as well as the main and most used acupoints in the therapy of this disease. Considering that acupuncture is meant to be an important tool both in the muscle strength restorement and in the pain control and inflammation, it enable the patient to attain a locomotor activity enhancement, an increasment of the joint movement angle, and a consequent life quality improvement, together with a lower cost, shorter recovering time and decreasing of surgical risks and post-surgical complications. Despite the low number of trials focused on the use ENCICLOPÉDIA BIOSFERA, Centro Científico Conhecer - Goiânia, v.14 n.26; p.1466 2017
\end{abstract}


of veterinary acupuncture in the treatment of dogs' hip dysplasia it's been registered that the local coxofemoral joint acupoints are the most widely used and allow the induction of the repairmen processes in an objective and properly effective way, above all when stimulated by simple needling and gold implants acupuncture methods.

KEYWORDS: Canines. Joint diseases. Traditional Chinese Medicine.

\section{INTRODUÇÃO}

A Medicina Tradicional Chinesa (MTC), que tem na acupuntura um de seus ramos, foi quase toda desenvolvida por meio da observação das leis da natureza e centrada para o tratamento do homem. Porém, com a importância dos animais na sociedade agrária, impulsionaram-se crescentes estudos e adaptações dos conceitos da MTC em modelos animais, chegando ao desenvolvimento da Medicina Veterinária Tradicional Chinesa (MVTC) (SCOGNAMILLO-SZABÓ; BECHARA, 2010).

O termo acupuntura foi firmado por jesuítas em visita a China durante o século XVII e é originário das palavras em latim acus=agulha e pungere=puncionar. Trata-se de uma terapia reflexa na qual estímulos nociceptivos, provocados pela introdução de finas agulhas, desencadeiam respostas em outras aéreas do organismo, permitindo atingir efeitos terapêuticos e homeostáticos que levam a promoção, manutenção e recuperação da saúde (SCOGNAMILLO-SZABÓ; BECHARA, 2010).

A acupuntura pode ser empregada no tratamento de diversas patologias, principalmente as alterações musculoesqueléticas, tendo como grande destaque o tratamento da displasia coxofemoral (DCF), uma afecção ortopédica de caráter hereditário que resulta em desenvolvimento ou crescimento anormal de uma ou ambas as articulações coxofemorais (PERRUPATO; QUIRINO, 2014). Dessa forma, objetivou-se com este estudo reunir informações a respeito do emprego da acupuntura veterinária na DCF em cães, assim como, identificar quais os principais métodos de acupuntura e os principais acupontos utilizados na terapêutica desta patologia.

\section{DISPLASIA COXOFEMORAL}

A displasia coxofemoral (DCF) é uma afecção ortopédica de caráter hereditário que resulta em desenvolvimento ou crescimento anormal de uma ou ambas as articulações coxofemorais. Caracteriza-se pela disparidade do crescimento entre os tecidos moles e a estrutura óssea da articulação, gerando vários graus de frouxidão articular, instabilidade e má formação da cabeça femoral e do acetábulo. (MIQUELETO et al., 2013; KING, 2017).

Como resultado da má formação das articulações coxofemorais, há um estresse anormal das estruturas de suporte, implicando em alterações inflamatórias que levam a maior produção de líquido sinovial e consequente aumento da pressão intra-articular (KYRIAZES, 2016). O espessamento da cápsula articular e do ligamento redondo, estão associados a frouxidão e instabilidade articular, favorecendo a ocorrência de subluxações ou luxação completa da cabeça femoral, que levam a ocorrência de micro fraturas acetabulares craniais, resultando em remodelação da cabeça do fêmur e acetábulo, arrasamento da cavidade acetabular e arredondamento das bordas (LIMA; PEREIRA, 2015; KING, 2017). 
Cães displásicos podem demonstrar sinais da dor através de mudanças de comportamento que podem ser percebidas pela tensão muscular, flexão brusca do membro quando manipulado, vocalização, agressão, entre outros aspectos clínicos que podem variar de acordo com a idade do animal. (ROCHA et al., 2013).

Nos animais jovens, o início dos sintomas é súbito, sendo observada flacidez articular, dor na flexão e extensão da articulação, rigidez ao se levantar, diminuição da atividade e claudicação intermitente ou continua (KYRIAZES, 2016). Já na fase adulta, os sinais clínicos são progressivos, normalmente ocultos e típicos de doença articular degenerativa (DAD). Geralmente demonstram crepitação articular, redução da amplitude de movimentos e claudicação, especialmente após exercícios prolongados. A flacidez articular pode não ser tão percebida em virtude da estabilização da articulação, associada ao espessamento da cápsula articular, deposição de osteófitos e fibrose periarticular (MIQUELETO et al., 2013).

\section{DIAGNÓSTICO}

O diagnóstico baseia-se na história detalhada da progressão da doença, achados clínicos e radiográficos (LIMA; PEREIRA, 2015). Testes específicos podem ser utilizados para avaliar a dor, a função articular e a integridade de seus componentes, dentre eles, podemos citar: os testes de Ortolani, Barlow e Bardens, avaliação da claudicação, testes de estação bípede, abdução com rotação externa e do iliopsoas, (ROCHA et al., 2013; KYRIAZES, 2016).

De acordo com as normas do Colégio Brasileiro de Radiologia Veterinária (CBRV), uma avaliação radiográfica preliminar pode ser feita a partir dos 12 meses de idade, porém apenas aos 24 meses podem ser realizadas avaliações definitivas. Para assegurar a boa qualidade das imagens radiográficas, é obrigatória a contenção química do paciente, mediante a utilização de associações farmacológicas capazes de proporcionar o perfeito relaxamento do animal (CBRV, 2013).

Toda a pelve e as articulações fêmoro-tíbio-patelares do paciente devem estar presentes na imagem. $\mathrm{O}$ animal deve ser posicionado em decúbito dorsal com os membros pélvicos em extensão, paralelos entre si e em relação a coluna vertebral, com uma leve rotação interna para sobrepor as patelas aos sulcos trocleares, tomando cuidando para que essas apareçam posicionadas entre os côndilos femorais. (CBRV, 2013; FCl, 2006).

O diagnóstico é confirmado de acordo com as evidencias radiográficas de subluxação ou luxação e DAD, incluindo a formação de osteófitos periarticulares, esclerose subcondral do bordo acetabular, remodelamento e aplanamento da cabeça e colo femoral, linha de "Morgan", arrasamento acetabular e aumento da densidade dos tecidos moles periarticulares (LIMA; PEREIRA, 2015).

\section{CLASSIFICAÇÃO DA DISPLASIA COXOFEMORAL E ESCALA DE DOR}

De acordo com o Colégio Brasileiro de Radiologia Veterinária e critérios da Federação Cinológica Internacional (FCI, 2006), a DCF pode ser classificada em 5 graus (CBRV, 2013). Com isso, afim de estimar o estado de dor do animal, levando em consideração aspectos comportamentais e respostas a estímulos de dor descritos respectivamente em escalas propostas pela Universidade do Estado do Colorado e escala descritiva simples, correlacionadas aos diferentes graus da DCF, constituímos o quadro abaixo. 
QUADRO 1 - Classificação da DCF e sua correlação com a escala de dor.

\begin{tabular}{|c|c|c|c|c|}
\hline GRAU & CLASSIFICAÇÃO & CARACTERÍSTICAS DA ARTICULAÇÃO & $\begin{array}{l}\text { PSICOLÓGICO E } \\
\text { COMPORTAMENTAL }\end{array}$ & $\begin{array}{l}\text { ESCALA } \\
\text { DE DOR }\end{array}$ \\
\hline A & $\begin{array}{l}\text { Articulações coxofemorais } \\
\text { normais }\end{array}$ & $\begin{array}{l}\text { - Cabeça femoral e o acetábulo são congruentes; } \\
\text { - Ângulo acetabular, segundo Norberg, é de aproximadamente } 105^{\circ} \text {. }\end{array}$ & Sem alterações & Sem dor \\
\hline B & $\begin{array}{l}\text { Articulações coxofemorais } \\
\text { próximas do normal }\end{array}$ & $\begin{array}{l}\text { - Cabeça femoral e o acetábulo são ligeiramente incongruentes e o } \\
\text { ângulo acetabular, segundo Norberg, é de aproximadamente } 105^{\circ} \text {, } \\
\text { ou; } \\
\text { - O centro da cabeça femoral se apresenta medialmente à borda } \\
\text { acetabular dorsal e a cabeça femoral e o acetábulo são } \\
\text { congruentes. }\end{array}$ & $\begin{array}{l}\text { - Confortável quando descansando; } \\
\text { - Feliz, contente; } \\
\text { - Interessado ou curioso pelo } \\
\text { ambiente; } \\
\text { - Não apresenta sensibilidade à } \\
\text { palpação. }\end{array}$ & Dor leve \\
\hline $\mathrm{C}$ & Displasia coxofemoral leve & $\begin{array}{l}\text { - Cabeça femoral e o acetábulo são incongruentes; } \\
\text { - Ângulo acetabular, segundo Norberg, é de aproximadamente } 100^{\circ} \\
\text { ou há um ligeiro achatamento da borda acetabular craniolateral, ou } \\
\text { ambos; } \\
\text { - Poderão estar presentes irregularidades ou pequenos sinais de } \\
\text { alterações osteoartrósicas da margem acetabular cranial, caudal ou } \\
\text { dorsal ou na cabeça e colo femoral. }\end{array}$ & $\begin{array}{l}\text { - Claudicação (+) } \\
\text { - Parece desconfortável quando } \\
\text { descansando; } \\
\text { - Levemente inquieto; } \\
\text { - Distrai-se facilmente; } \\
\text { - Expressão facial preocupada; } \\
\text { - Não demonstra interesse pelo } \\
\text { ambiente ou em interagir com } \\
\text { pessoas; } \\
\text { - Reage à palpação. }\end{array}$ & $\begin{array}{c}\text { Dor } \\
\text { moderada }\end{array}$ \\
\hline D & $\begin{array}{l}\text { Displasia coxofemoral } \\
\text { moderada }\end{array}$ & $\begin{array}{l}\text { - Incongruência evidente entre a cabeça femoral e o acetábulo; } \\
\text { - Sinais de subluxação; } \\
\text { - Ângulo acetabular, segundo Norberg, é de aproximadamente } 95^{\circ} \text {; } \\
\text { - Presença de achatamento da borda crânio-lateral ou sinais } \\
\text { osteoartrósicos, ou ambas. }\end{array}$ & $\begin{array}{l}\text { - Claudicação (++); } \\
\text { - Desconfortável ao sentar; } \\
\text { - Desconfiado, vocalizando; } \\
\text { - Protege o membro afetado e pode } \\
\text { reagir agressivamente; } \\
\text { - Mostra-se pouco disposto a se } \\
\text { movimentar; } \\
\text { - Reação dramática à palpação. }\end{array}$ & Dor severa \\
\hline $\mathrm{E}$ & $\begin{array}{l}\text { Displasia coxofemoral } \\
\text { severa }\end{array}$ & $\begin{array}{l}\text { - Alterações displásicas da articulação coxofemoral evidentes; } \\
\text { - Sinais de luxação ou distinta subluxação; } \\
\text { - Ângulo acetabular, segundo Norberg, menor que } 90^{\circ} ; \\
\text { - Evidente achatamento da borda acetabular cranial; } \\
\text { - Deformação da cabeça femoral ou outros sinais de osteoartrose. }\end{array}$ & $\begin{array}{l}\text { - Decúbito permanente; } \\
\text { - Vocalização constante; } \\
\text { - Potencialmente irresponsivo aos } \\
\text { estímulos do ambiente; } \\
\text { - Difícil de ser distraído; } \\
\text { - Pode reagir agressivamente à } \\
\text { palpação. }\end{array}$ & $\begin{array}{l}\text { Dor muito } \\
\text { severa }\end{array}$ \\
\hline
\end{tabular}

Fonte: FCI, 2006; GAYNOR; MUIR, 2009; CBRV, 2013.

\section{TRATAMENTO}

Levando em consideração que nenhum método é capaz de restaurar completamente a articulação displásica, objetiva-se com o tratamento, controlar a dor e inflamação, reduzir as alterações degenerativas, melhorar a condição articular, restabelecer a capacidade motora e dessa forma, melhorar a qualidade de vida do paciente (ROCHA et al., 2013).

A seleção da terapia adequada leva em consideração a idade, condição de saúde do paciente, sinais clínicos, achados radiográficos e as condições financeiras do proprietário (LIMA; PEREIRA, 2015). Casos leves podem ser controlados através de meios conservadores, que envolvem repouso, controle de peso por meio do manejo alimentar e exercícios controlados, associados a fisioterapia, administração de anti-inflamatórios não esteroidais e condroprotetores (KIRBKY; LEWIS, 2012; LIMA; PEREIRA, 2015).

Em quadros mais avançados, o tratamento conservador não proporciona de forma eficiente os resultados esperados, sendo necessária a realização de técnicas cirúrgicas. No entanto, esse tipo de procedimento depende de fatores como, condições fisiológicas adequadas, idade e porte para seu sucesso. Sem contar que representam métodos invasivos, possuem altos custos e podem oferecer altos riscos e diversos inconvenientes pós-cirúrgicos ao paciente (LOPES, et al., 2011).

Nesse contexto, a acupuntura tem se mostrado importante ferramenta no tratamento da DCF. Pode ser utilizada tanto em conjunto à métodos de tratamento ocidentais convencionais, quanto isoladamente, já que possui efeitos comprovados 
no restabelecimento da força muscular, controle da dor e inflamação. Além de dispensar o uso de fármacos, evitar problemas pós-operatórios e possuir menor custo econômico quando comparada aos métodos cirúrgicos (HULEA; CRISTINA, 2012).

\section{ACUPUNTURA}

O termo acupuntura foi firmado por jesuítas em visita a China durante o século XVII e é originário das palavras em latim acus=agulha e pungere=puncionar. No vocábulo chinês é definido como Zhen jiu, onde possui um sentido mais abrangente: literalmente "agulha-moxabustão "(SCOGNAMILLO-SZABÓ; BECHARA, 2010).

É uma técnica terapêutica milenar baseada em conhecimentos teóricoempíricos da cultura oriental. Tem na sua filosofia a ideia de equilíbrio, tanto em se tratando de funções orgânicas quanto na relação do corpo com o meio ambiente. Isso deve-se a suas bases filosóficas, que estão contidas nas teorias gerais do Taoísmo (Yin Yang e Teoria dos Cinco Elementos) (SCOGNAMILLO-SZABÓ; BECHARA, 2010), inicialmente criadas para a compreensão e interpretação dos fenômenos naturais. Representando a base conceitual tanto da MTC quanto da MVTC (XIE; PREAST, 2012).

Do ponto de vista cientifico, trata-se de uma terapia reflexa na qual os estímulos nociceptivos, provocados pela introdução de finas agulhas em pontos anatômicos específicos denominados acupontos, desencadeiam respostas em outras aéreas do organismo, permitindo atingir efeitos terapêuticos e homeostáticos que levam a promoção, manutenção e recuperação da saúde (SCOGNAMILLOSZABÓ; BECHARA, 2010).

\section{MÉTODOS DE ACUPUNTURA}

Diversos métodos de estimulação dos acupontos podem ser empregados na Medicina Veterinária. Dentre os principais, podemos citar: acupuntura por agulhamento simples, método que consiste na inserção de finas agulhas metálicas na superfície corporal do paciente (ROMANA, 2013); moxibustão, utiliza o calor da queima do material produzido com folhas de Artemisia vulgaris, afim promover o aumento da velocidade de circulação energética nos canais de energia (NÚÑES; RODRÍGUEZ, 2014); laserpuntura, utiliza baixa potência de laser para estimular os acupontos (XIE; PREAST, 2012); eletroacupuntura, que consiste na aplicação de impulsos elétricos conduzidos por eletrodos ou agulhas previamente inseridas nos acupontos, afim de promover estímulos em diferentes intensidades e frequências (BRAGA; SILVA, 2012); e os implantes, procedimento cirúrgico-ambulatorial, onde, implantes confeccionados de diversos tipos de materiais, principalmente o ouro, são implantados em tecidos próximos aos acupontos, permitindo a estimulação prolongada ou até mesmo constante desses (LOBO JUNIOR, 2012).

Podemos citar ainda, métodos menos difundidos, tais como: o uso de ventosas, que utiliza a pressão negativa de dentro de um recipiente com válvulas de borracha ou aquecidos, para sugar a pele, afim de estimular o tecido local e terminações nervosas (NÚÑES; RODRÍGUEZ, 2014); acuinjeção, corresponde a aplicação de substâncias injetáveis nos acupontos, como: soluções salinas ou água destilada (aquapuntura); glicose e vitaminas; autólogos de sangue (hemopuntura); veneno de abelha (apipuntura); injeção de ar (pneumoacupuntura); injeção de fármacos (farmacopuntura) e injeção de células-tronco (MARX et al., 2014). 


\section{APLICAÇÕES DA ACUPUNTURA}

Dentre os objetivos terapêuticos gerais da acupuntura, podemos citar: a recuperação motora; restauração da força muscular; redução da tensão muscular e espasmos; melhora do fluxo sanguíneo; regulação das funções orgânicas, endócrinas, autônomas e mentais; modulação da imunidade e ativação de processos regenerativos; além de promover efeitos que inibem a inflamação e geram analgesia (MCGOWAN; GOOF; STUBBS, 2011).

Em meio as afecções que podem ser submetidas ao tratamento com a acupuntura, podemos citar: os distúrbios do sistema urinário; gastrintestinais; cardíacos; reprodutivos; hepáticos; patologias relacionadas ao sistema neurológico, tais como, epilepsias, doenças do disco intervertebral, paraplegias/paraparesias, sequelas de cinomose, acidentes vasculares cerebrais, síndrome de Horner idiopática; doenças dermatológicas causadas por reações alérgicas ou desordens autoimunes; e principalmente as alterações musculoesqueléticas, tendo como grande destaque o tratamento da DCF (LOBO JUNIOR, 2012).

\section{MECANISMO DE AÇÃO NA DISPLASIA COXOFEMORAL}

A ciência ainda não foi capaz de descrever todos os reais efeitos da acupuntura, no entanto, sabe-se que estes ocorrem por associações de mecanismos neurológicos e humorais complexos (MASSONE, 2011), e que seus efeitos ocorrem de maneira diferente, dependendo dos acupontos selecionados, do método de estimulação que será empregado e da duração desses estímulos (COLLAZO, 2012).

Os acupontos estão presentes em locais anatômicos específicos e apresentam características físicas, fisiológicas e histológicas distintas quando comparadas a estruturas adjacentes (ALVARENGA et al., 2014). Próximos a estes, estão presentes capilares sanguíneos, vasos sanguíneos e linfáticos, ampla concentração de terminações nervosas sensoriais associadas ao sistema nervoso autônomo, feixes e plexos nervosos, células imunomoduladoras, glândulas, estruturas de tecidos moles como fibras musculares, colágeno e outros tecidos conjuntivos (ZHANG et al., 2012).

Apesar da grande parte dos estudos estarem voltados para a analgesia, sabese que a acupuntura também possui efeitos significativos em mecanismos antiinflamatórios, alterações na dinâmica da circulação sanguínea e relaxamento muscular (ROMANA, 2013). Nesse viés, tornando a acupuntura, uma ferramenta indiscutivelmente importante no tratamento da DCF em cães.

As agulhas de acupuntura possuem propriedades biofísicas com as quais desenvolvem um potencial elétrico na ponta. Quando inseridas e manipuladas, desencadeiam estímulos que agem sobre as estruturas presentes nos acupontos, alterando o potencial da membrana celular, acarretando o desencadeamento do potencial de ação e consequente condução dos estímulos nervosos, que resultam tanto em efeitos locais quanto sistêmicos (COLLAZO, 2012; LINDSEY et al., 2014).

Os estímulos produzidos pela acupuntura produzem efeitos pré-sinápticos espinhal, por meio da ativação de fibras aferentes de nervos periféricos (Tipo II e/ ou A-delta) (COLLAZO, 2012). Os impulsos nervosos gerados pela acupuntura ascendem via fascículo ventrolateral, ativando três níveis do sistema nervoso: medula espinhal; substância cinzenta pareaquedutal; núcleo magno da rafe e o complexo hipotálamo-hipófisario. Quando cada um desses níveis é estimulado, desencadeia-se uma cascata química que resulta na liberação de neurotransmissores, neuromoduladores e outras substâncias (COLLAZO, 2012). 
Nessa cascata, os peptídeos opióides endógenos (encefalina, endorfinas e dinorfinas) e a serotonina (5-hidroxitriptamina) são os principais responsáveis por promover o bloqueio da transmissão da dor no sistema nervoso central (LINDSEY et al., 2014).

Além desses, acredita-se que a substância $P$, por meio da ação de proteases dos mastócitos provavelmente regula a ação do gene da calcitonina das terminações nervosas e a sua atividade vasodilatadora, e que a estimulação adicional de fibras C, através dos mecanismos do "gate control theory", também resulta em interrupção da dor e relaxamento muscular (LINDSEY et al., 2014).

O estimulo das agulhas de acupuntura ao atingir o hipotálamo, induzem ainda a liberação de beta-endorfina na corrente sanguínea e do hormônio adrenocorticotrópico (ACTH) na hipófise, responsáveis respectivamente pelo bloqueio da dor e por estimular a córtex adrenal para que libere cortisol na circulação, mediando assim os efeitos anti-inflamatórios da acupuntura (ALVARENGA et al., 2014).

Os estímulos produzidos pela acupuntura, podem ainda, alterar a dinâmica da circulação sanguínea local, por meio da indução de uma inflamação asséptica, que estimula a liberação de serotoninas e adrenalina e consequentemente provocará vasodilatação, e assim, maior perfusão tecidual local, permitindo que a musculatura afetada seja melhor irrigada, que toxinas metabólicas, como lactato, sejam diluídas e transportadas através de vasos sanguíneos e linfáticos, agindo de maneira eficaz no restabelecimento do tônus muscular perdido durante 0 processo patológico (ROMANA, 2013).

\section{DESENVOLVIMENTO}

Afim de identificar quais métodos de acupuntura e acupontos oferecem melhores resultados. Nessa revisão, são apresentados 12 estudos que avaliam o emprego da acupuntura no tratamento de cães com DCF. Esses, são descritos a seguir e apresentados de forma resumida no Quadro 2.

Gülanber et al. (1995) em estudo realizado em 15 cães, tinham como objetivo avaliar as vantagens e desvantagens do uso da acupuntura como tratamento para a DCF. Para isso, utilizaram a eletroacupuntura e implantes de ouro na estimulação dos acupontos: VB29; VB30; VB34; B29; B49; E36 e pontos Ashi (pontos dolorosos). Entretanto, os autores não justificam o uso de tais acupontos na terapêutica empregada. Ao fim do estudo os autores indicam a acupuntura como um método alternativo ou auxiliar no tratamento da DCF.

Em estudo prospectivo, randomizado, duplo-cego e controlado, Fink et al. (2001) avaliaram a eficácia do tratamento da osteoartrite do quadril através do método de acupuntura por agulhamento simples. Sessenta e sete animais foram divididos em dois grupos, onde o grupo 1 recebeu o emprego e manipulação tradicional da agulha, inseridos no acupontos VB30; VB34; VB31; E40; B37; B54 e seis pontos Ashi. Já o grupo 2 teve as agulhas colocadas longe do posicionamento clássico e sem manipulação. Os autores citam que os acupontos foram selecionados seguindo as recomendações presentes na literatura. Porém, não justificam o uso dos mesmos. Concluem ainda, que ambos os grupos de tratamento possuem efeitos positivos na terapêutica da DCF.

Hielm-bjorkman et al. (2001) tiveram como objetivo em seu estudo, avaliarem em ambiente controlado, randomizado e duplo-cego um grupo de trinta e oito cães com DCF. Para 19 desses animais, usaram-se implantes de ouro nos acupontos: 
VB29, VB30 e B54, além desse, outros três pontos extras, não especificados pelos autores, que possuíam alta condutividade e estavam presentes em quatro articulações do quadril, também foram estimulados com a finalidade de otimizar os resultados. Entretanto, os autores não justificaram o uso de tais acupontos. Ao fim de 24 semanas esse estudo não observou diferença significante entre os grupos.

Bührer (2002) apresenta em seu trabalho dois casos clínicos de DCF, onde os pacientes receberam tratamento por meio do método de acupuntura por agulhamento simples. Em ambos os casos, foram realizadas diversas sessões de acupuntura com diferentes combinações dos seguintes acupontos e suas respectivas indicações: R7 - elimina edema nos membros posteriores e é utilizado para fraquezas em geral; B11 - fortalece os ossos, retarda a degeneração dos ossos; B26 - fortalece a parte inferior das costas, remove obstruções do meridiano e é indicado em lombalgias inferior crônica; B60 - fortalece o Qi dos rins, relaxa os tendões e músculos; BP2 - usado em doenças febris sem transpiração; R25 indicado em anorexia e fluxo de linfa melhorado; E31 - indicado em casos de síndrome da atrofia e displasia coxofemoral e fortalece o membro posterior; E32remove a obstrução dos meridianos e é indicado para displasia coxofemoral; E36 fortalece o corpo e resolve o edema; E40 - resolve a fleuma e a umidade e elimina o calor; IG4 - promove analgesia, alivia a ansiedade e estimula o parto; IG11 - ponto imunoestimulante; P9 - estimula a circulação sanguínea e influencia o pulso; VB29 indicado em doenças da articulação do quadril, dores e paralisia na extremidade posterior e remove obstrução do meridiano; VB39 - indicado em doenças da articulação do quadril e inchaço da extremidade; VB34 - regula a mobilidade das articulações e é o ponto mestre dos tendões e articulações; F3 - relaxa músculos e tendões ; F8 - indicado em dores na área dos órgão pélvicos e na articulação medial do joelho; VG14- dispersa o vento e circula o Yang Qi do corpo; VG20 - fortalece a função ascendente do baço; R3 - tonifica o Qi dos rins, restaura o Yin Qi e nutre o Qi e o sangue; C7 - estimula a circulação sanguínea e influencia o pulmão, ponto de sedação do coração, problemas funcionais do coração e possui efeito calmante em inquietude geral. Além desses acupontos já citados o autor associou em algumas sessões, métodos de craniopuntura para motricidade e cromopuntura nos olhos. Ao fim do tratamento os resultados foram satisfatórios e os animais puderam retornar a suas atividades diárias.

Sawaya (2007) indicou em seu trabalho o uso dos acupontos VB29, VB30, B54, B40, R1, B67, BP2, VG2, B23, VB34, B60 para o tratamento de artrite do quadril, selecionados após exame clinico e de acordo com a sensibilidade dos acupontos na palpação. Porém, não justificou a utilização desses acupontos. Contudo, sugere o emprego da acupuntura em quadros agudos, subagudos e crônicos de artrite do quadril.

No trabalho apresentado por Scogmillo-Szabo et al. (2010), foi utilizado protocolo de tratamento com acupuntura e posterior utilização de implantes de ouro para manutenção do quadro clínico atingido. Os acupontos estimulados através do método de acupuntura por agulhamento simples neste trabalho foram respectivamente indicados para efeitos regionais nos membros posteriores $(\mathrm{BH}$ e B40), tônico geral (VG1), patologias da articulação coxofemoral (VB30 e B36), ação regional em patologias musculares e da articulação coxofemoral (VB34), fortalecimento de ligamentos e tendões (F3), ação sobre o metabolismo de estruturas ósseas (B23) e ação sobre o metabolismo de ligamentos e tendões (B18). No momento da utilização do método de implante de ouro os acupontos estimulados foram: B54, VB29 e VB30, juntamente com mais 6 pontos Ashi. A paciente 
apresentou resposta favorável a acupuntura por agulhamento simples, com remissão da dor e reabilitação locomotora, após o emprego de implantes de ouro, os resultados permaneceram estáveis durante o período de acompanhamento.

No estudo apresentado por Lima et al. (2011), 13 animais de diversas raças, de ambos os sexos e diferentes faixas etárias, atendidos no Hospital Veterinário Universitário da Universidade Federal do Píaui (UFPI) com sinais clínicos e exame radiográfico compatíveis com a DCF, foram submetidos a protocolo de tratamento por acupuntura que utilizou em todos os pacientes os acupontos: VB29; VB30; B23; B52; B40; B36; B60; R3 e VG4, visando padronizar os acupontos no tratamento dessa enfermidade como protocolo. Entretanto, os autores não justificaram a utilização dos mesmos. Após a primeira sessão com intervalo semanal, $100 \%$ dos animais obtiveram melhora no quadro clínico e ao final do estudo, 12 animais (92\%) apresentaram recuperação total da sintomatologia.

Lopes et al. (2011) relataram o caso clinico de um cão da raça Boxer com diagnóstico de DCF bilateral de grau moderado. O paciente foi impossibilitado de realizar o procedimento cirúrgico para tratamento da DCF por apresentar alterações no hemograma, com isso, optou-se pelo uso do método de acupuntura por agulhamento simples com o intuito de buscar um meio complementar para a melhora clínica do animal. Os acupontos estimulados no presente trabalho foram: VG20; R3; B60; E36 e VB30, não sendo justificada sua escolha. Imediatamente após a primeira sessão foi observada diminuição do desconforto e ao fim do tratamento constatou-se o aumento do ângulo da articulação coxofemoral e sinais clínicos ausentes.

Em um ensaio clínico prospectivo, duplo-cego, controlado com placebo, realizado por Jaeger et al. (2012), foi descrito o método de implantes de ouro 24 quilates na articulação coxofemoral de cães de diversas raças (28 raças), de ambos os sexos, idade variadas entre 1-8 anos e com diferentes graus de DCF, afim de avaliar os efeitos e complicações desse tratamento. Para isso, foram implantados dois fragmentos de ouro em cada um dos acupontos selecionados: VB29, VB30, B54 e dois Ashi. Porém a utilização desse, não foi justificada pelos autores. Ao fim, concluíram que o método de implante de ouro é seguro e facilmente aplicável, no entanto pode resultar em agravamento da dor durante as duas primeiras semanas pós implante.

Lobo Junior (2012), expõe dois casos de animais diagnosticados para DCF. Onde, um recebeu tratamento com implantes de ouro enquanto o outro passou por sessões de acupuntura por agulhamento simples e posteriormente optou-se pela utilização dos implantes de ouro. Para os dois casos, foram selecionados os mesmos acupontos, os quais foram utilizados segundo as seguintes indicações: VB30 - fortalece a coluna vertebral da região lombar e membros posteriores, remove obstruções de Qi, relaxa tendões e músculos e dispersa a umidade; VB54 - fortalece a coluna lombar e dissolve umidade e calor; VB29 - fortalece a coluna lombar, relaxa os tendões e músculos e dispersa umidade e calor; F3 - melhora a função do fígado e circulação do sangue; $\mathrm{BH}$ - mantém e regula o Yang Qi do organismo, dispersa o vento e umidade e relaxa os tendões e músculos; B23 - usado para tonificar o rim e o Yin geral do organismo; B18 - usado para tonificar o fígado e retirar a estagnação do órgão. Nos dois casos os animais recuperaram-se totalmente.

Em trabalho realizado por Perrupato e Quirino (2014) foi relatado o atendimento de um cão da raça Labrador Retriever, com sinais de DCF grave e sequelas de cinomose no Hospital Veterinário da Unigran (Centro Universitário da 
Grande Dourados). Na terapêutica indicada para o tratamento da DCF foram utilizados os seguintes acupontos: VB29, VB30, BH, R3 e B60, porém os autores não justificaram o uso de tais acupontos. Ao fim do tratamento observou-se melhora dos sinais clínicos, tais como diminuição da dor e aumento do ângulo de movimento da articulação, melhora nas atividades locomotoras, maior tolerância a exercícios e maior desenvolvimento dos músculos pélvicos.

Marx et al. (2014) propuseram em estudo realizado na Universidade Luterana do Brasil, a injeção de células-tronco mesenquimais isoladas a partir autólogos de frações vascular estromal e tecido adiposo alogênico nos acupontos VB29, VB30 e B54, afim de avaliar a eficácia desse método no tratamento de cães com DCF. Porém, não justificaram a utilização desses acupontos. Concluiu-se com o experimento que o tratamento se mostrou eficaz na melhora clinica dos animais presentes no estudo.

De acordo com suas teorias, a acupuntura compreende uma terapia individualizada, onde, cada indivíduo possui uma abordagem e respostas terapêuticas distintas para uma mesma patologia (SCOGNAMILLO-SZABÓ et al., 2010, LIMA et al., 2011). Durante esta revisão foram relatados o emprego da acupuntura para a estimulação dos diversos acupontos, no entanto, notou-se que os acupontos mais comumente empregados foram exatamente três pontos locais da articulação coxofemoral, os quais formam um triângulo ao redor da cabeça do fêmur: B54, VB29 e VB30.

O acupunto B54 é chamado de Zhi bian, e tem como ação, beneficiar a região dorsal e a micção. O acuponto VB29, conhecido como Ju liao, é muito utilizado no tratamento da DCF devido sua ação geral na região do quadril e por sua proximidade da articulação. Tem como objetivo, remove obstruções do canal, tratar a dor no quadril ou na região dorsal dos membros posteriores, fortalece a coluna lombar e relaxar tendões e músculos. O acuponto VB30 chamado de Huan tiao, é utilizado por ser o encontro dos canais da vesícula biliar e bexiga, como esses canais passam "em cima" da articulação sua ação é rápida e eficaz. É indicado no fortalecimento da coluna vertebral da região lombar e membros posteriores, em dores no quadril e para o relaxamento de tendões e músculos. Quando utilizado em combinação com o acuponto VB29, torna-se muito eficaz (LOBO JUNIOR, 2012). 
QUADRO 2 - Estudos do emprego da acupuntura na displasia

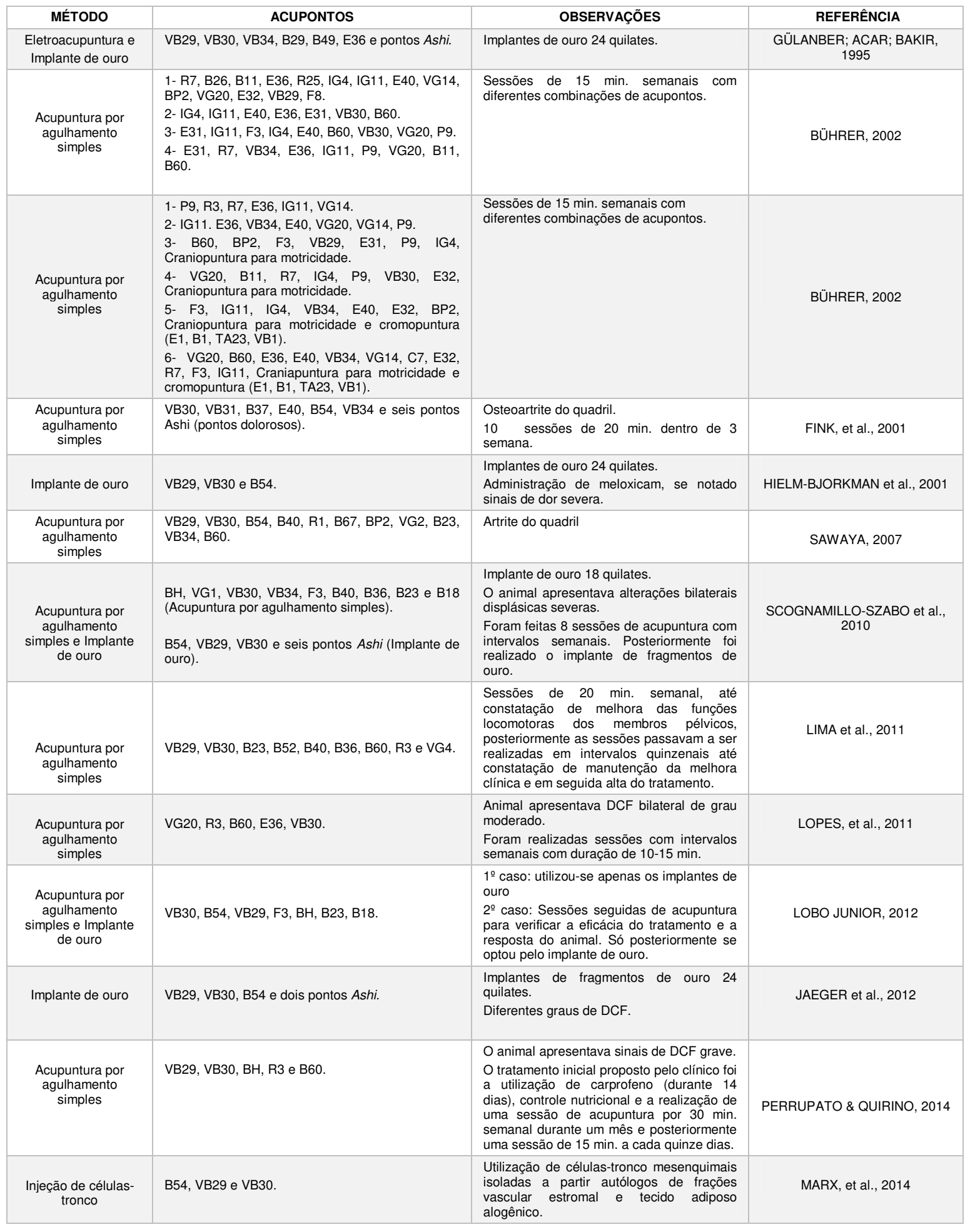

Verificou-se nesta revisão que os métodos de acupuntura mais empregados no tratamento da DCF em cães foram: acupuntura por agulhamento simples e implantes de ouro. Porém, por ser uma patologia degenerativa que necessita de tratamento clinico contínuo o implante de ouro se mostra como melhor opção, já que permite a estimulação constante dos acupontos, dispensando as várias sessões do método de acupuntura por agulhamento simples. 
O ouro é um material extremamente resistente a corrosão, sendo considerado biocompatível e quando implantado emite pequenas cargas elétricas positivas que neutralizam as cargas negativas induzidas por respostas inflamatórias, diminuindo ou eliminando a dor e prevenindo alterações artríticas na articulação (SCOGNAMILLO-SZABÓ et al., 2010). Talvez o uso prévio do método de acupuntura por agulhamento simples pode resultar em melhores respostas do implante de ouro, com mostrado nos estudos de Nejrup et al. (2008), já que provavelmente essa associação pode ter resultados expressivos na resposta retardada dos implantes de ouro, que como relatado em alguns estudos podem geram agravamento da dor, desconforto, claudicação e rigidez, durante alguns dias pós implante, como consequência de uma resposta dolorosa do tecido ainda não compreendidas (SCOGNAMILLO-SZABÓ et al., 2010; JAEGER et al., 2012).

\section{CONSIDERAÇÕES FINAIS}

Apesar de ainda ter sua eficácia questionada, talvez pelo pouco conhecimento dos profissionais a respeito dos benéficos do seu emprego, a acupuntura sem dúvida representa um método terapêutico de grande relevância para a Medicina Veterinária. Mostrando-se importante ferramenta no restabelecimento da força muscular, controle da dor e inflamação, melhora das atividades locomotoras e do ângulo de movimento da articulação, permitindo maior tolerância a exercícios e consequentemente melhoria da qualidade de vida de cães com DCF. Quando aplicada por profissionais capacitados não apresenta efeitos colaterais e nem contraindicações, podendo ser aplicada isoladamente ou em conjunto a outros métodos de tratamento ocidentais. Quando comparado aos métodos convencionais de tratamento, possui melhor custo econômico, requer menor tempo de recuperação e evita os riscos cirúrgicos e complicações pósoperatórias.

Mesmo com pouca quantidade de estudos direcionados ao emprego da acupuntura veterinária na displasia coxofemoral em cães, notou-se que os acupontos, mais empregados foram: B54, VB29 e VB30. Exatamente acupontos locais da articulação coxofemoral, concordando com o fato de que os principais acupontos a serem estimulados em patologias musculoesqueléticas devem ser aqueles próximos a área afetada, já que estes induzem processos de reparação de forma objetiva e efetiva na articulação.

Talvez, por ser o método mais difundido na Medicina Veterinária, o método de acupuntura por agulhamento simples foi o mais empregado nos estudos revisados, porém, de acordo com os estudos, o implante de ouro mostrou ser a melhor opção, já que permite a estimulação constante dos acupontos, dispensado as várias sessões requeridas pelo método de acupuntura por agulhamento simples. Contudo, novas pesquisas continuam a ser produzidas e novos métodos promissores estão surgindo, a exemplo do método de injeção de células-tronco, que vem mostrando-se eficaz no tratamento da DCF e podem vir a ser de grande valia para o futuro.

\section{REFERÊNCIAS}

ALVARENGA, T.F; AMARAL, C.G; STEFFEN, C.P. Ação da acupuntura na neurofisiologia da dor: revisão bibliográfica. Revista Amazônia Science \& Health. v. 2. n. 4 , p. 29-36. Out/Dez, 2014. Disponível em: <http://ojs.unirg.edu.br/index.php/2/article/view/530>. 
BRAGA, N.S; SILVA, A.R.C. Acupuntura como opção para analgesia em veterinária. PUBVET, Londrina, V. 6, N. 28, Ed. 215, Art. 1435, 2012. Disponível em: < http://pubvet.com.br/uploads/efcd3f42e23b2f34111b39193ae41fe7.pdf>.

BÜHRER, P.C. Displasia coxofemoral canina: alternativa de tratamento com acupuntura. 2002. 109 f. Monografa (Graduação em Medicina Veterinária) Universidade Tuiuti do Paraná, Curitiba, PR. Disponível em: $<$ http://tcconline.utp.br/wp-content/uploads/2013/08/DISPLASIA-COXOFEMORALCANINA.pdf>.

COLÉGIO BRASILEIRO DE RADIOLOGIA VETERINÁRIA. Normas do Colégio Brasileiro de Radiologia Veterinária (CBRV) para avaliação da displasia coxofemoral em cães. São Paulo, 2013. Disponível em: < http://www.abrv.org.br/arquivos/normas-do-colegio.pdf>. Acesso em: 20.03.17.

COLLAZO, E. Fundamentos actuales de la terapia acupuntural. Revista Sociedad Española del Dolor. v. 19. n. 6, p. 325-331, nov - dez, 2012. Disponível em: http://scielo.isciii.es/scielo.php?script=sci_arttext\&pid=S113480462012000600007\&l $\mathrm{ng}=\mathrm{pt} \&$ tlng $=\mathrm{es}>$.

FEDERATION CYNOLOGIQUE INTERNATIONALE (FCI). Requisitos de la FCI para la detección de la displasia de cadera. Copenhague, Dinamarca, mar, 2006. Disponível em: < http://www.fci.be/es/Displasia-de-cadera-y-codo-162.html>. Acesso em: 24.03.17.

FINK, M.G.; KUNSEBECK, H.; WIPPERMAN, B.; GEHRKE, A. Non-specific effects of traditional Chinese acupuncture in osteoarthritis of the hip. Complementary Therapies in Medicine. v. 9, p. 82-88, 2001. Disponível em: <https://doi.org/10.1054/ctim.2001.0442>. DOI: 10.1054/ctim.2001.0442.

GAYNOR, J.S; MUIR III, W.W. Manual de controle da dor em medicina veterinária. 2. ed. São Paulo: Medvet, 2009.

GÜLANBER, E.G.; ACAR, S.E.; BAKIR, B. Köpeklerde kalça displazisinin acupunkturla tedavisi. Veteriner Cerrahi Dergisi. v. 1, n. 1, p. 21-25, 1995. Disponível

em:<http://www.ggacademia.com/FileUpload/bs483714/File/kopeklerde_kalca_displa zisi_akupunktur_acupuncture_canine_hip_dysplasia.pdf>.

HIELM-BJORKMAN, A.; RAEKALLIO, M.; KUUSELA, E.; MARKKOLA, A.; TULAMO, R-M. Double-blind evaluation of implants of gold wire at acupuncture points in the dog as a treatment for osteoarthritis induced by hip dysplasia. The Veterinary Record, p. 452-456, oct. 2001. Disponível em: < http://dx.doi.org/10.1136/vr.149.15.452>. DOI: 10.1136/vr.149.15.452.

HUELA, C.I; CRISTINA, R.T. Acupuncture as a Therapeutic Tool in Health Disorders in Animals: a Review. Scientific Papers: Animal Science and Biotechnologies, v. 
45, n. 2, p. 166-177, 2012. Disponível em: < http://spasb.ro/index.php/spasb/article/view/340/221>.

JAEGER, G.T.; OYVIND, S.; MORTEN, D.; LARS, M. Gold Bead Implantation in Acupoints for Coxofemoral Arthrosis in Dogs: Method Description and Adverse Effects. Animals. v. 2, p. 426-436, 2012. Disponível em: < http://www.mdpi.com/2076-2615/2/3/426/htm>. DOI: 10.3390/ani2030426.

KING, M.G. Etiopathogenesis of Canine Hip Dysplasia, Prevalence, and Genetics. Veterinary of North America: Clinics Small Animal. 2017. Disponível em: <http://www.sciencedirect.com/science/article/pii/S0195561617300220?via\%3Dihub> . DOI: https://doi.org/10.1016/j.cvsm.2017.03.001.

KIRKBY, K.A.; LEWIS, D.D. Canine Hip Dysplasia: Reviewing the Evidence for Nonsurgical Management. Veterinary Surgery. v.41, p.2-9, 2012. Disponível em: <http://www.svsvet.com/downloads/Hip-Dysplasia.pdf>.

DOI:10.1111/j.1532950X.2011.00928.x.

KYRIAZIS, A. Canine hip dysplasia. Part I: Aetiopathogenesis \& diagnostic approach. Hellenic Journal of Companion Animal Medicine. v. 5, n. 1, p. 37, $2016 . \quad$ Disponível em: <http://hjcam.hcavs.gr/images/vol5iss1/izs_vol5_issue1_canine-hip-dysplasia.pdf>.

LIMA, B.B; DIAS, F.G.G; PEREIRA, L.F. Diagnóstico e tratamento conservador da displasia coxofemoral em cães. Revista Investigação Medicina Veterinária. v. 14, p. 78-82, 2015. Disponível em: <http://publicacoes.unifran.br/index.php/investigacao/article/view/834>.

LIMA, V.B.S.; KLEIN, R.P.; QUESSADA, A.M.; SOUZA, J.M. Acupuntura no tratamento de displasia coxofemoral em cão. Universidade Federal do Piauí (UFPI), $2011 . \quad$ Disponível em: <http://leg.ufpi.br/20sic/Documentos/RESUMOS/Modalidade/Vida/a42a596fc71e178 28440030074d15e74.pdf>.

LINDSEY, M.F.; NEARY, S.; SHARROCK, J.; RYCHEL, J.K. Acupuncture for Analgesia in Veterinary Medicine. Topics in Companion Animal Medicine. 2014. Disponível em: <http://www.sciencedirect.com/science/article/pii/S1938973614000051>. DOI: https://doi.org/10.1053/j.tcam.2014.03.001.

LOBO JUNIOR, J.E.S. Acupuntura na pratica clínica veterinária. São Caetano do Sul, SP: Interbook, 2012.

LOPES, K.R.G. MOURA, M.; MOURI JR, S.C.; RODRIGUES, M.R.; BABINSKI, M.A. Displasia coxofemoral em cães da raça boxer: diagnóstico semiológico, radiográfico e tratamento clinicamente eficaz pela acupuntura. Acta Scientiae Medica Online. v. 4, n. $1, \quad$ p. 22-32, 2011. Disponível em: < 
https://www.yumpu.com/pt/document/view/12834233/acta-scientiae-medica-on-linevol-41-22-32-2011-et-al-1997->.

MCGOWAN, C.M; GOFF, L; STUBBS, N. Fisioterapia animal: avaliação, tratamento e reabilitação de animais. São Paulo: Roca, 2011.

MARX, C.; SILVEIRA, M.D.; SELBACH, I.; SILVA, A.S.; BRAGA, L.M.G.M.; CAMASSOLA, M.; NARDI, N.B. Acupoint injection of autologous stromal vascular fraction and allogeneic adipose-derived stem cells to treat hip dysplasia in dog. Stem Cells International. v. 2014, n. p. 1 - 6, Porto Alegre, RS, Aug. 2014. Disponível em: $\quad$ https://www.hindawi.com/journals/sci/2014/391274/abs/>. $\quad$ DOI: http://dx.doi.org/10.1155/2014/391274.

MASSONE, F. Anestesiologia Veterinária: farmacologia e técnica: texto e atlas colorido. ed. 6, Rio de janeiro: Guanabara Koogan, 2011.

MIQUELETO, N.S.M.L.; RAHAL, S.C.; AGOSTINHO, F.S.; SIQUEIRA, E.G.M.; ARAÚJO, F.A.P.; MENESES, A.M.C.; EL-WARRAK, A.O. Displasia coxofemoral e a análise cinemática. Veterinária e Zootecnia, v. 20, n. 2, p. 9 - 15, jun. 2013. Disponível em: <http://www.fmvz.unesp.br/rvz/index.php/rvz/article/view/170>.

NEJRUP, K.; OLIVARIUS, N.F.; JACOBSEN, J.L.; SIERSMA, V. Randomised controlled trial of extraarticular gold bead implantation for treatment of knee osteoarthritis: a pilot study. Clinical Rheumatology. v. 27, n., p. 1363-1369, 2008. Disponível em: < https://www.ncbi.nlm.nih.gov/pubmed/18500437>. DOI: 10.1007/s10067-008-0918-9.

NÚÑEZ, H.M.R.; RODRÍGUEZ, G.B. Métodos de manipulación en la medicina tradicional asiática. Santiago de Cuba, Cuba: Medisan, v. 18, n. 5, p. 696, 2014. Disponível em: <http://scielo.sld.cu/scielo.php?pid=S102930192014000500013\&script=sci_arttext\&tlng=pt>.

PERRUPATO, T. F.; QUIRINO A. C. T. Acupuntura como terapia complementar no tratamento de displasia coxofemoral em cães - relato de caso. Revista Ciências Veterinária e Saúde Pública, v. 1, n. 2, p. 141-145, 2014. Disponível em: <http://ojs.uem.br/ojs/index.php/RevCiVet/article/view/24176>. DOI: http://dx.doi.org/10.4025/revcivet.v1i2.24176

ROCHA, L.B.; TUDURY, E.A.; ROEHSIG, C. BARAÚNA, D.; CHIORATTO, R.; ARAÚJO, F.P.; KEMPER, B. Denervação articular coxofemoral em cães com doença articular degenerativa secundária à displasia. Ciências Animal Brasileira. v.14, n.1, p. 120-134, jan.- mar. 2013. Disponível em: <http://189.126.110.61/cab/article/view/5946>. DOI: http://dx.doi.org/10.1590/10.5216/cab.v14i1.3528.

ROCHA, S.P.; BENEDETTO, M.A.C.; FERNANDEZ, F.H.B.; GALLIAN, D.M.C. A trajetória da introdução e regulamentação da acupuntura no Brasil: memórias de desafios e lutas. Ciências Saúde Coletiva. v.20, n.1. Rio de Janeiro.jan.2015. 
Disponível

<http://www.scielo.br/scielo.php?script=sci_arttext\&pid=S141381232015000100155\& Ing=pt\&tlng=pt>. DOI: http://dx.doi.org/10.1590/1413-81232014201.18902013.

ROMANA, R.C. Acupuntura, electroacupuntura, moxibustión y técnicas relacionadas en el tratamiento del dolor. Revista Sociedad Española del Dolor, v. 20, n. 5, p. 263-277.Sep-Oct.2013. Disponível em: < http://scielo.isciii.es/scielo.php?script=sci_arttext\&pid=S1134-80462013000500006>. DOI: http://dx.doi.org/10.4321/S1134-80462013000500006.

SAWAYA, S. Physical and alternative therapies in the management of arthritic patients. Veterinary Focus. v. 17, n. 3, p. $37-42,2007$. Disponível em: < http://www.edliny.com/Encyclopedy/Veterinary\%20Focus/173/7.pdf>.

SCOGNAMILLO-SZABÓ, M.V.R.; BECHARA, G.H. Acupuntura: histórico, bases teóricas e sua aplicação em Medicina Veterinária. Ciência Rural, Santa Maria, v.40, n.2, p. 491-500, fev. 2010. Disponível em: < http://www.scielo.br/scielo.php?script=sci_arttext\&pid=S0103-84782010000200040 > . DOI: http://dx.doi.org/10.1590/S0103-84782010005000004.

SCOGNAMILLO-SZABÓ, M.V.R.; SOUSA, N.R.; TANNÚS, L.; CARVALHO, F.S.R. Acupuntura e implante de fragmentos de ouro em pontos de acupuntura e pontos gatilho para o tratamento de displasia coxo-femoral em Pastor Alemão. Acta Scientiae Veterinariae. v. 38, n. 4, p. 433-448, 2010. Disponível em: <http://www.ufrgs.br/actavet/38-4/PUB\%20938.pdf>.

XIE, H.; PREAST, V. Medicina veterinária tradicional chinesa: princípios fundamentais. 1 ed. São Paulo: MedVet, 2012.

ZHANG-JIN, Z; XIAO-MIN, W; GRAINNE, M.M. Neural Acupuncture Unit: A new concept for interpreting effects and mechanisms of acupuncture. Evidence-Based Complementary and Alternative Medicine, v. 2012, n. p. 1-23, 2012. Disponível em: < https://www.hindawi.com/journals/ecam/2012/429412/abs/>. $\quad$ DOI: http://dx.doi.org/10.1155/2012/429412. 